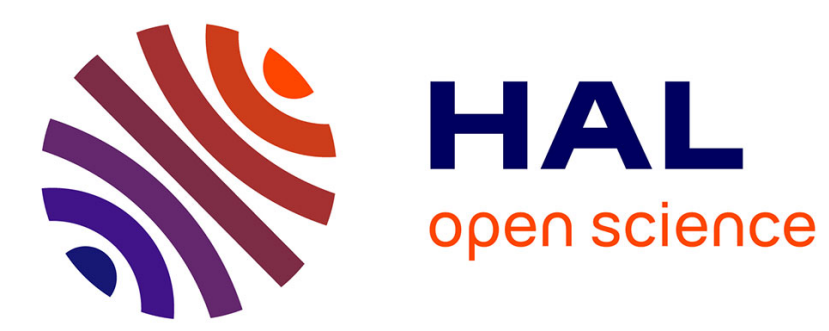

\title{
A Protocol to Address User Behavior in the Eco-Design of Consumer Products
}

Emmanuelle Cor, Peggy Zwolinski

\section{To cite this version:}

Emmanuelle Cor, Peggy Zwolinski. A Protocol to Address User Behavior in the Eco-Design of Consumer Products. Journal of Mechanical Design, 2015, Special Issue: User Needs and Preferences in Engineering Design, 137 (7), pp.10. 10.1115/1.4030048 . hal-01242790

\section{HAL Id: hal-01242790 \\ https://hal.science/hal-01242790}

Submitted on 15 Dec 2015

HAL is a multi-disciplinary open access archive for the deposit and dissemination of scientific research documents, whether they are published or not. The documents may come from teaching and research institutions in France or abroad, or from public or private research centers.
L'archive ouverte pluridisciplinaire HAL, est destinée au dépôt et à la diffusion de documents scientifiques de niveau recherche, publiés ou non, émanant des établissements d'enseignement et de recherche français ou étrangers, des laboratoires publics ou privés. 


\author{
Emmanuelle Cor ${ }^{1}$ \\ G-SCOP, \\ Université Grenoble Alpes, \\ 46 Avenue Félix Viallet, \\ Grenoble Cedex 138031 , France \\ e-mail: emmanuelle.cor@grenoble-inp.fr \\ Peggy Zwolinski \\ G-SCOP, \\ Universite Grenoble Alpes, \\ 46 Avenue Félix Viallet, \\ Grenoble Cedex 1 38031, France \\ e-mail: peggy.zwolinski@grenoble-inp.fr
}

\section{A Protocol to Address User Behavior in the Eco-Design of Consumer Products}

Today, it is difficult to integrate the use phase optimization of consumer products into eco-design methodologies. Current eco-design approaches are in fact mainly focused on improving the technological performance of products while it has been proven that users behavior plays an important role in the overall environmental performances of products. This paper deals with the need to address the notion of user experience and behavior in the design process of today's low-complexity consumer products in order to improve their environmental performance. The research protocol presented in this paper is a new ecodesign approach in six steps that can be used by designers to support eco-design decisions and integrate user behavior parameters into design activities. The first step consists in the identification of critical environmental aspects in use and usage drifts potential of the product. Steps two, three, and four support designers in the analysis of the use phase for the selection of efficient design for sustainable behavior (DfSB) strategies to be implemented on the product. Finally, steps five and six aim to test the selected strategies with product-in-use observations. Life cycle assessment (LCA) approach is used for the evaluation of the environmental benefits of the strategies. To illustrate this work, a case study of a coffee maker is described together with the eco-design solutions chosen for this product. The solutions reflect strategies targeting DfSB. [DOI: 10.1115/1.4030048]

\section{Introduction and Context of the Work}

Over the last decades, increasing competition on the consumer products market has promoted innovation and an extensive diversification of offers proposed to consumers [1]. However, this diversification has induced a considerable number of environmental problems stemming from the over-use of raw materials and energy [2]. Indeed, new consumption modes are not in line with sustainable development principles [3], which is why it is necessary to rethink products and offer design processes in order to limit their associated environmental impacts.

The environmental optimization of consumer product manufacturing, distribution, and end-of-life phases has been a focal point of research over the last few decades [4]. However, the environmental impacts of most of these products, especially electrical and electronic appliances, stem mainly from their use phase [5]. Indeed, this life cycle phase accounts for $60-80 \%$ of the global environmental impacts of such products [6]. Existing eco-design strategies targeting optimization of the use phase are mainly based on improving product technological performances: substitution of technologies, process efficiency, architecture transformation [7], and other technological variants. Given the growing complexity of these products and new technologies, the apparition of usage drifts during the product usage may be observed. A usage drift can be defined as improper use of the product compared with the average use scenario defined by the manufacturer. It becomes therefore difficult to believe in efficient product use without some form of progressive assistance for the user [8]. User-related needs and variables are not being sufficiently integrated into current eco-design decisions with a view to reduce the environmental impacts associated with the product use phase.

1.1 Use Phase Integration Into Current Eco-Design Approaches. Current eco-design approaches aim to integrate environmental aspects into product design and development right

\footnotetext{
${ }^{1}$ Corresponding author.

Contributed by the Design Automation Committee of ASME for publication in the Journal of Mechanical Design. Manuscript received September 14, 2014; final manuscript received March 5, 2015; published online May 19, 2015. Assoc. Editor: Carolyn Seepersad.
}

from the early design phase. The aim is to reduce the environmental impacts of products throughout their entire life cycle [9]. Decisions made during the early design phase can considerably influence the future environmental impacts of products [10,11]. ISO standard 14,062 [9] divides the product life cycle into five main phases: raw material acquisition, manufacturing, trade and delivery, use/maintenance, and end of life (including disposal, reuse, and recycling). The product use phase starts when the product leaves the store where it is sold and ends when it is considered as waste [12].

There are currently insufficient specifications pertaining to this use phase in standards and literature. For Sauer et al. [13], this is mainly because manufacturers have little control over this life cycle use phase and because product use scenarios are so diverse. Because of the difficulty entailed in defining this life cycle phase, consumer product eco-design approaches have mainly focused on finding design solutions to optimize other life cycle phases [4]. "Design for X" strategies have been setup by industry and researchers mainly to improve the manufacturing and end-of-life phases in terms of environmental impacts $[14,15]$. However, it has been demonstrated that the use phase of household appliances has an important impact on the environment compared with other phases [6]. Some design tools that do include the use phase dimension in the eco-design of consumer products have nevertheless been developed over time by researchers and industry [16] The various methods established mainly aim to meet regulatory energy efficiency targets for energy related products $[17,18]$. According to the work of Kuijer and De Jong [19] on usercentered sustainable design, use phase eco-design solutions can be broken down into the following three categories:

- Technological innovations (improving product efficiency via technology)

- Use behavior innovations (designing products to influence user behavior)

- Practice innovations (changing business models-e.g., car sharing)

The argument put forward in this paper is that use-related environmental impacts can only be decreased when these three aspects of product design are simultaneously considered. Today, priority 
should be given to the integration of user behavior dimensions into product development while solutions should be combined with technological innovations and sustainable business models.

1.2 From User Behavior Characterization to DfSB Strategies. Studying user behavior can provide designers with important information for the design of products with lower use-related environmental impacts [20]. Lidman et al. [21] define a proenvironmental behavior as the action of using an artefact in a way that has lower environmental impacts than the conventional way in which the artefact is used. Switching off electronic appliances, recycling waste generated by the use of a product, and limiting consumables required for use (electricity and water) are just some examples of pro-environmental behaviors. Identifying the variables influencing behavior during product usage can help designers understand how the right behavior might be promoted through product design. Several models of user variables influencing behavior have been created to understand how to promote these behavioral changes. The theory of planned behavior developed by Ajzen [22] studies the relationships between attitude, subjective norms, perceived behavioral control, and behavior. Abrahamse et al. [23] in the sociopsychological research area identify other variables, which are also of importance when it comes to characterizing and understanding user behavior: the context of use of the product, societal factors, personal factors as well as habits, knowledge, and routines.

The product also plays an important role in shaping use-related practices involving the consumption of resources [19]. According to Srivastava and Shu [24], the improvement of environmental impacts during the use phase can be encouraged on a long-term basis through product design. DfSB is an emerging research field and activity where the aim is to reduce the environmental and social impacts of products by controlling users' interaction with them [25]. In fact, with the right design strategies (implementation of new features on the product, redesign of the product, information given to the user, etc.), designers can shape activities relating to product use. Many researchers have worked on the mechanisms employed in DfSB strategies in order to induce pro-environmental behavior [20]. Several practical examples of DfSB can be found in literature. One of these, the power aware cord [26], developed by the Interactive Institute, is an example of the direct feedback strategy. This wire is designed so that the user knows how much electricity is being drawn off the grid: the more current, the brighter the cord. Some studies on information-feedback methods for saving energy in households have proven that feedback and information systems support energy consumption reduction [27-29]. One of the main conclusions of Wood and Newborough's study [28] is that further attention needs to be given for the optimization of the design of feedbacks method in order to maximize the associated energy-saving potential. However, no global environmental assessments of these systems from a life cycle perspective have ever been proposed. Feedback systems have also been used frequently in the design of automobiles with the aim of limiting fuel consumption by steering user behavior (e.g., the BMW eco pro mode). In the case of feedback strategies, users remain in control of their behavior: the product gives them information that can induce behavioral change. In other cases, design intervention strategies are chosen to better control behavior such as clever design strategies [30]. These aim to decrease environmental impacts through design solutions without changing user behavior. Automatic standby modes implemented on household appliances are an example of clever design strategies. These strategies can also be compared with the functionality matching strategies developed by Wever et al. [31], which aim to bring user needs in line with product functionalities. However, Lilley [25] demonstrated that design strategies developed to steer user behavior without restricting user interactions with the product seem to be more widely accepted than those exerting greater control over the user. Several strategy classifications have been proposed in the literature. These depend on the extent to which the strategy intervenes with product use, the possible format of this intervention or the possible design strategy employed (Table 1). However, from these existing classifications, designers find it difficult to evaluate how efficient DfSB strategies really are from a life cycle perspective. Design solutions found to promote pro-environmental behavior and limit environmental impacts in use phase may cause impact transfers on other life cycle phases of the product (e.g., manufacturing and end of life phases) [8]. There is in fact a lack of integration of eco-design concepts (such as life cycle thinking and environmental analysis) in this DfSB research field in order to support the decisions taken during the design process for efficient implementation of DfSB strategies [32].

In response to the need described above, this paper presents a user-focused research protocol for low-complexity electrical consumer appliances. The overall aim is to improve the use-related environmental performance of this type of product. The protocol therefore sets out an eco-design methodology based on DfSB concepts. Using this protocol, designers can address use-related behavior in order to improve the environmental performance of consumer products during use. Moreover, the impact analysis of DfSB strategies realized by the LCA of a product's global life cycle provides designers with new information for the early design phase. Section 2 of this paper presents each step of the protocol and the methodological elements on which it is built. The implementation of this protocol is then detailed in Sec. 3 through a case study. Finally, the discussion and conclusion (Secs. 4 and 5) highlight the main findings of this research and the future work to be conducted on this topic.

\section{A Research Protocol to Address User Behavior in the Eco-Design of the Use Phase of Consumers Products}

The aim of this experimental protocol is to address user behavior and needs in the eco-design of the use phase of lowcomplexity electrical appliances. Based on a microlevel analysis of the use phase, the protocol helps to identify relevant DfSB strategies to be implemented on a product in order to reduce the environmental impacts stemming from the product's use by modifying user behavior. This involves:

- Identifying product usage drifts

- Analyzing user profiles

- Measuring use-related environmental performances in different design intervention strategies for sustainable behavior

- Identifying potential efficient design solutions

- Analyzing environmental impacts of chosen design solutions

The protocol is divided into six main steps. The first step consists in the identification of critical environmental aspects in use and usage drifts potential of the product. Steps two, three, and four support simultaneously designers in the analysis of the use phase for the selection of efficient DfSB strategies to be implemented on the product. Finally, steps five and six aim to test the selected strategies with product-in-use observations using a life cycle vision. The next parts describe each of these steps.

2.1 Step 1: Identification of Possible Usage Drifts on the Product. This first step aims to help designers identify the potential for implementing a DfSB strategy for the product in question. This step is based on a qualitative analysis of the use phase during which the designers provide a qualitative response to questions related to:

- Product functionality

- Product maintenance

- Environmental safety performance

- Consumption flows during the use phase (resources and wastes) 
The resulting output of this step is the identification of the main possible product usage drifts together with the significant environmental aspects associated with each drift. In the context of this study, a usage drift is characterized as a task performed by the user on the product that undermines its global environmental performance as calculated on the basis of the average use scenario. Improper maintenance, intensive product use, and inappropriate use of the product's functionalities are examples of usage drifts. The contribution of user behavior to the global environmental impacts of the use phase is qualitatively evaluated in this step. If the potential usage drifts represent a large share of the global environmental impacts incurred during use, the designers can go through to the next step of the protocol. If not, the designers must implement a clever design strategy or make technical improvements to the product in order to render its use more efficient. Therefore, some affirmations are unrelated to usage drifts and other focus on this particular aspect. The affirmations contained in the questionnaire can be adjusted according to the typology of product studied. A life cycle analysis (LCA) is coupled with the qualitative analysis to back up the results obtained. Following this first step, if a strong usage drifts potential has been found on the studied product, designers can go through the next three steps of the protocol, which aim to provide a deep analysis of the product use phase for the identification of relevant DfSB strategies to be implemented.

2.2 Step 2: Development of a Relationship Model Tying Product Design With User and Environmental Impacts During Product Use. Once the designers have identified potential for environmental gains during the product use phase by modifying user behavior, a representative model of the use phase is then developed. Several sociopsychological studies have proposed different representations of the variables influencing user behavior in order to understand how to promote behavioral changes [33,34]. The theory of planned behavior where the relationship between attitude, subjective norms, perceived behavioral control, and behavior is identified is one approach stemming from such studies [22]. Sauer et al. [8] used this theory to build a model of the relationship between user behavior and the environmental impacts of product use in order to examine ways of improving ecological user performance from a designer perspective. The context of use characterized by various parameters such as the environment of use, geographic conditions, the equipment available to the user, etc. [35] needs to be added to Sauer's model. In fact, applying

Table 1 Classification of DfSB strategies according to the objective and implementation format of the strategies [4]

\begin{tabular}{ll}
\hline \hline $\begin{array}{l}\text { DfSB strategy objective } \\
\text { Selvefors and coworkers [36] }\end{array}$ & \multicolumn{1}{c}{$\begin{array}{c}\text { Implementation format } \\
\text { Selvefors and coworkers [36] }\end{array}$} \\
\hline Increase knowledge & Adapted information \\
& Written information \\
& Oral information \\
& Demonstration \\
Engage & Comparative feedback \\
& Self-monitoring \\
& Social validation \\
& Objective to reach \\
Steer and spur & Competition \\
& Guilt \\
& Constraints \\
& Penalties \\
Motivation \\
Create attention & Persuasive technology \\
& Behavior steering \\
& Affordance \\
& Real time-feedback \\
& Personalized feedback \\
\hline
\end{tabular}

DfSB strategies to the product will modify the context of use thus influencing user use-related activities and reducing the environmental impacts of product use. It is, however, necessary to balance the environmental gains with the environmental losses due to the implementation of DfSB strategies on the product. The generic model developed in this work (Fig. 1) establishes the relationship between product design, user variables, context of use, use-related activities, and use-related environmental impacts. Based on this representation, designers can define the variables influencing this model more precisely for each typology of product. This step of the protocol helps designers to examine the influence of design intervention strategies on the product's context of use and on user behavior.

2.3 Step 3: Identification of the User Profile. To address user needs and preferences in the definition of design intervention strategies for sustainable behavior, it is necessary to perform a detailed analysis of the future users of the product studied $[8,23]$. In step 3 of this protocol, a questionnaire based on the literature study presented in 1.2 is addressed to potential users. This questionnaire is used to measure different user variables to help define the environmental sensitivity of users: environmental knowledge, environmental habits, and environmental attitude. These three variables have been selected to test different behavioral responses to DfSB strategies according to users' habits, knowledge, and attitude. Other variables of interest identified by designers could be measured in this step. Section 3.3 details more specifically how the questionnaire was developed for our case study and how the environmental profile of the users was determined according to the users' answers.

To summarize, this step helps designers to better identify the typology of users so that the relevant DfSB strategy can be implemented according to their profile. "Eco-sensitive" (ES) users are defined as those individuals who might, for example, obtain a high score for their environmental habits in the questionnaire while "non-eco-sensitive" (NES) users are those with a low score. It might therefore be supposed that different levels of DfSB intervention strategies need to be implemented for these two different user categories. The questionnaire needs to be disseminated to a wide and representative panel of users. Data collected are analyzed to separate the participants into the two groups (ES and NES) (cf., 3.3).

2.4 Step 4: Identification of DfSB Strategies to be Implemented and Tested on the Product. The objective of step 4 of this research protocol is to identify potential effective DfSB intervention strategies that can then be implemented and tested on the product studied. The choice of strategies to be implemented is based on:

- The level of strategy intervention on user behavior (engage, steer and spur, create attention, etc.) according to the company's objectives $[23,36]$

- The implementation format (written information, ecofeedback, etc.) $[30,25]$

- The product typology (functionalities, usage drifts, etc.)

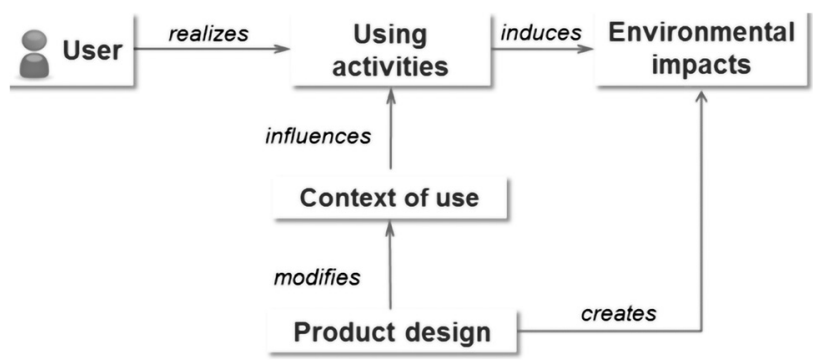

Fig. 1 General model of the relationships between users, product design, and environmental impacts during use 
- The resources available for the tester (in terms of costs, time, human resources, etc.)

Designers may find the classifications and examples of DfSB strategies reported in literature useful when it comes to choosing those to be tested on the product studied (cf., Table 1). Several strategies can be tested to identify the most viable one. Once the strategies have been selected, their implementation on the product is done through prototyping.

2.5 Step 5: Product-In-Use Observations. Step 5 is the core step of this protocol. Each design strategy for sustainable behavior chosen in step 4 is tested by users in a real-life context and product-in-use observations are made. The product-in-use observation is an interactive, observational method designed to "capture peoples' behavior in real-life contexts;" it provides an "account of the behavior surrounding a product or activity" [37]. This step aims to analyze individual behavior in relation to the instrumented product and identify potential user behavior modifications compared with a noninstrumented product. The observations must be performed in an "as-at-home" environment in order to observe at best habitual behavior in relation to the product. During this phase, the environmental impacts of the product as it is used must be measured and usage drifts recorded (by video and based on the product prototyping). Long-term product-in-use observations are preferable to short-term observations. The level of user acceptance of each strategy also has to be evaluated with a postexperiment questionnaire.

2.6 Step 6: Results Analysis and Implementation in the Design Process. In the last step of the protocol, the results of the observations are analyzed. This will help with the selection of the right design solution from a life cycle point of view and according to the user's profile. A life cycle analysis should be carried out to evaluate the potential environmental benefits produced by the design intervention strategies and stemming from a change in the user's behavior. The global environmental impacts of the initial product (noninstrumented) can be calculated using LCA software as shown in the below equation:

$$
\mathrm{IE}_{\text {global }}=\mathrm{IE}_{\mathrm{use}}+\mathrm{IE}_{\mathrm{rme}}+\mathrm{IE}_{\mathrm{m}}+\mathrm{IE}_{\mathrm{d}}+\mathrm{IE}_{\mathrm{eol}}
$$

where IE is environmental impact, rme is raw material extraction phase, $\mathrm{m}$ is manufacturing phase, $\mathrm{d}$ is distribution phase, and eol is end of life phase.

When design intervention strategies are applied to the product, the environmental impacts incurred by use of the product are supposed to decrease $\left(\Delta \mathrm{IE}_{\mathrm{use}}<0\right)$ while the environmental impacts associated with other life cycle phases may increase $\left(\Delta \mathrm{IE}_{\mathrm{rme}, \mathrm{m}, \mathrm{d}, \mathrm{eol}}>0\right)$. The global variation in environmental impacts according to whether the product is instrumented with a DfSB strategy or not is calculated as shown in the below equation:

$$
\Delta \mathrm{IE}_{\text {global }}=\Delta \mathrm{IE}_{\text {use }}+\Delta \mathrm{IE}_{\text {rme }}+\Delta \mathrm{IE}_{\mathrm{m}}+\Delta \mathrm{IE}_{\mathrm{d}}+\Delta \mathrm{IE}_{\text {eol }}
$$

If the variation in global environmental impacts is significantly negative $\left(\Delta \mathrm{IE}_{\text {global }}<0\right)$, then the design intervention strategy applied to the product can be considered to have a positive effect on the environmental performance of the product. Designers can also use the analysis of the user's level of acceptance of the strategy to decide which strategy should be implemented on the product.

In order to illustrate this protocol, a case study on a coffee maker is presented in the next part of this paper.

\section{Case Study: Application of the Protocol to the Redesign of a Coffee Maker}

An espresso coffee maker functioning with ground coffee and a filter holder was chosen for this case study (Delonghi EC 150).
The machine is a simple espresso maker frequently used in European households. The product has three functioning modes that are manually controlled by one button: a "turn off" mode, a "preheating" mode, and a "pouring coffee" mode. This type of product was selected for this study because the use phase of a coffee maker contributes significantly to the product's global environmental impacts [38]. Moreover, the chosen model proved to generate many usage drifts that could be limited with design intervention strategies for sustainable behavior (cf., 3.1). For these reasons, the Delonghi EC 150 has been selected in the context of this study for a redesign project, using the six steps protocol described previously. The description of the application of this eco-design approach step by step on the coffee maker is presented below:

3.1 Step 1: Identification of Potential Usage Drifts for the Coffee Maker. In order to identify potential coffee maker usage drifts, a checklist based on the drifts presented in the eco-design pilot tool [39] was developed. This checklist provides the design team with several affirmations related to the use phase of the product. As they go through the list, the designers are required to indicate their level of agreement. Some of the affirmations help them to identify potential product usage drifts. Others help them to identify technological improvements that could be made in the early design phase to improve the environmental performance of the product during use. An excerpt of this questionnaire is presented in Fig. 2 with examples of the affirmations. If the design team disagrees with an affirmation, a score of two points is awarded. If it agrees, 0 points are awarded for the affirmation. If the designers remain undecided, only one point is awarded. This scoring system helps to transfer qualitative information into quantitative information. The higher the score is, the higher the potential for improving the product. A score of $0 \%$ in one of the four main use phase categories (cf., 3.1) indicates that the product is already optimized for that category.

With this scoring system, it is possible to display a graph showing potential improvements to product use environmental performance either based on the user (implementation of DfSB strategies) or on the product's technology (integration of more efficient components, product architecture, etc.).

Figure 3 displays the results of the coffee maker use phase qualitative analysis. The main coffee maker usage drifts identified through this qualitative analysis are as follows:

- A high level of coffee consumption inducing electricity, water and ground coffee consumption, and waste production

- Poor maintenance of the coffee maker (no descaling) inducing energy consumption and a shorter lifetime

- Coffee maker left on after the coffee is made (can induce up to $30 \%$ extra energy consumption over the global life cycle of the product [40])

This qualitative assessment therefore helps the designers to identify critical environmental aspects of the coffee maker during the use phase as well as potential usage drifts. Following on from these results, DfSB strategies can be applied to this product to improve its environmental performance during use. In this case study, the designers targeted DfSB strategies able to reduce the energy consumption flow during use as this flow contributes highly to the product's environmental impacts during use. This decision was backed up by a review of the LCA study on the coffee maker [38]. The water flow and coffee flow could also have been the focus of the DfSB strategies.

3.2 Step 2: Development of the Model of Use-Related Activities for the Coffee Maker. A model of the relationship between users, environmental impacts during use, and the context of use was developed for the coffee maker based on the methodology outlined in Sec. 2.2 and Sauer's model [8]. In order to create this model, the following hypotheses were taken into account: 


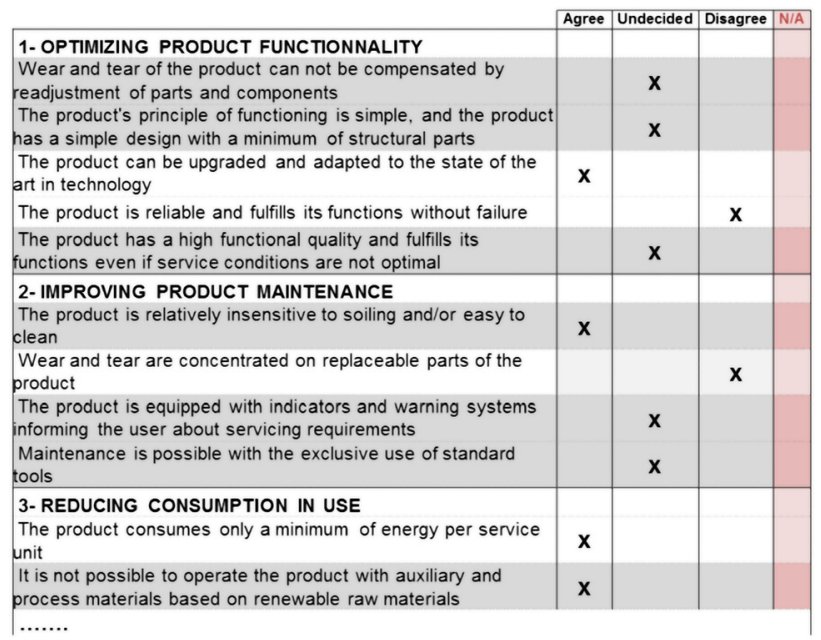

Fig. 2 Questionnaire based on the checklist from the ecodesign pilot [39] to support designers for the identification of usage drifts

- User behavior is influenced by the environmental profile of the user (and the variables measured)

- The context of use (defined in Sec. 2.2) is modified by the design solutions implemented on the product. This contextual modification leads to a change in user behavior and thus influences activities pertaining to the product's use.

- Environmental impacts during use are induced by userelated activities and are thus correlated with use-related behavior

Figure 4 illustrates the model developed for the coffee maker. The next steps of the protocol aim to validate the hypotheses made in step 2 with the definition of the correlation between design intervention strategies, context of use, user behavior, and environmental impacts during use.

3.3 Step 3: Identification of the User Profile. An online questionnaire was developed to measure different user variables in order to define the environmental sensitivity of users: environmental knowledge, environmental habits, and environmental attitude.

- Environmental knowledge: A ten-item questionnaire was developed to evaluate the general environmental knowledge of users. In the questionnaire, respondents were asked to agree or disagree to general affirmations on major environmental issues. One item, for example, claimed that "global ice melting can lead to the rise of sea level and provokes flooding." When answering the questionnaire, users had to indicate how confident they were in their answer using a fivepoint Likert scale. The final score of this questionnaire varied between -10 and 10 , with 10 representing a high level of environmental knowledge.

- Environmental habits: A 25-item questionnaire was developed to evaluate the general environmental habits of the users. Ten items out of the 25 targeted users' habits in relation to their coffee maker at home. The other questions are related to users' general habits regarding waste recycling, water consumption, use of domestic appliances, etc. One item, for example, stated "I collect and recycle used paper." The respondents were asked to define the frequency with which they performed the action in question on a six-point Likert scale (always-never). A maximum score of 25 suggested strong pro-environmental habits.

- Environmental attitude: The new environmental paradigm (NEP) scale revised method [41] consisting of 15 items (eight

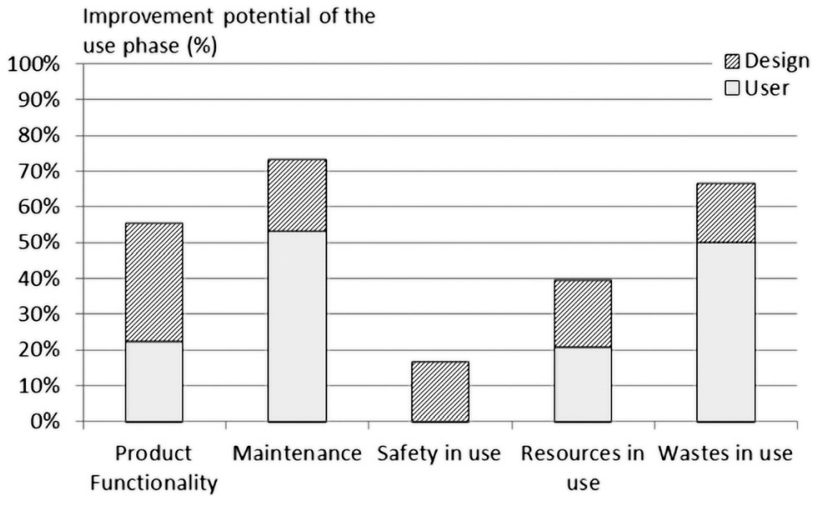

Fig. 3 Results of the qualitative analysis of possible coffee maker usage drifts

protraits and seven constraints) was used to measure users' tendency to accept a certain environmental vision. The maximum score for this questionnaire is 75. Dunlap et al. [41] have demonstrated that a high score on the NEP scale is correlated with a pro-environmentalist attitude. This scale was used in this protocol to indirectly measure the environmental attitude of the users.

Definition of users' eco-profiles: A panel of 155 individuals using an espresso coffee maker at home answered the online questionnaire. The data collected from the questionnaires were then analyzed using a principal component analysis that grouped the participants according to their environmental sensitivity profiles. The analysis led to a graphical identification of two groups of participants. The first cluster corresponds to people who had a high score for environmental knowledge, habits, and environmental attitude. Out of this first cluster, eighteen people with the best questionnaire scores were selected to represent the ES group. The second cluster corresponds to participants who obtained low scores in the questionnaire (on knowledge habits and attitude). Out of this second cluster, eighteen people with the lowest questionnaire scores were selected to represent the NES group. These 36 participants were considered to represent the average profile of European espresso coffee maker owners (profile established on the basis of a study performed on 70 espresso coffee maker owners in France and Spain) (67\% females; 33\% males: 19\% aged between 22 and 32; 31\% aged between 33 and 43; $19 \%$ between 44 and 54; and $31 \%$ between 55 and 65 ). This experiment could not be performed on the 155 online questionnaire respondents as this would have required too much time and too many resources ( $1 \mathrm{hr}$ for the experiment and 15 euros per participant). For a larger panel to strengthen the analysis, more resources would be required.

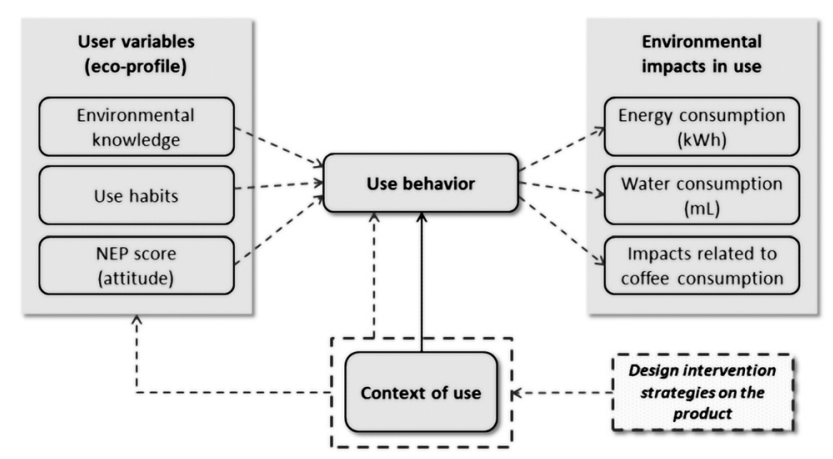

Fig. 4 Impact of the integration of design intervention strategies for the product on the model of relationships between user variables, context of use, and environmental impacts during use for the coffee maker 
3.4 Step 4: Identification of DfSB Strategies to be Implemented and Tested on the Product. Four design intervention strategies were chosen to be tested during this experiment:

- Eco-feedback (S1) facilitates for users to make environmentally sound decisions by giving immediate feedback (in our case on energy consumption) about use behavior [30]

- Objective to reach (S2) sets to users a performance goal (in our case, to consume less than $10 \mathrm{Wh}$ for making a coffee) for pro-environmental behaviors [23]

- Forcing technology (or forced functionality) (S3) is the design of the product in a way that hinders unsustainable behaviors (in our case, the implementation of an automatic switch off for the coffee maker) [31]

- Written information (S4) that gives instruction for sustainable behavior (in our case, a reminder to the user to turn off the maker).

Figure 5 presents how these strategies have been implemented on the coffee maker. These four strategies were chosen because they have a different level of influence on user behavior (respectively, inform, steer, spur, and force the user). Moreover, they were relatively easy to implement and be tested on this type of product. In order to measure the energy consumed during use in the context of the experiment, each prototype was equipped with sensors to measure the energy consumed and the time needed to make the coffee. The aim was to verify the efficiency of the strategies by measuring these variables during the experiment.

3.5 Step 5: Product-In-Use Observations. The experiment was divided into five main phases (The numbers circled in Fig. 6 represent each of these phases for more clarity):

Phase 1: The panel of participants (36 people) was divided into two groups $(2 \times 18$ people $)$ depending on their environmental sensitivity profile as outlined in the procedure in Sec. 3.3.

Phase 2: After a phase during which the users learned to use the coffee maker, the participants in both groups, ES and NES, were asked to make a coffee as they would do at home on a noninstrumented coffee maker. This coffee is considered as the "reference coffee" in the rest of this paper. The global electricity consumption per coffee (Wh) was measured during observation phase 1 (O1, Fig. 6)

Phase 3: The two groups were each divided randomly into three subgroups of six people. All the people in the same subgroup made two coffees on a coffee maker instrumented with S1, S3, or S4 (eco-feedback, persuasive technology, or written information). In this way, each design intervention strategy was tested by 12 people: 6 ES and 6 NES. The global electricity consumption per coffee (Wh) was measured (O2, Fig. 6).

Phase 4: The two subgroups that tested the coffee-maker instrumented with $\mathrm{S} 1$ were asked to make two more coffees using a coffee maker instrumented with S2: eco-feedback + objective. As stated above, strategy S2 could only be tested together with S1. The global electricity consumption per coffee (Wh) was measured (O3, Fig. 6).

Phase 5: At the end of the experiment, all the participants were asked to fill in a postexperimental questionnaire. The aim of this questionnaire was to evaluate users' perception of the efficiency, utility, and intrusiveness of the intervention strategies they tested as well as their acceptance of these strategies.

Each phase was video-recorded to capture behavior during use and identify usage drifts. Sensors installed on each prototype were used to measure use-related environmental impacts (energy consumption) and the time needed for each task (cf., 3.4).

3.6 Step 6: Results Analysis and Implementation in the Design Process. Environmental performances measured during the product-in-use observation have then to be analyzed and balanced with the environmental losses due to the adding of technologies on the prototypes. Step 6 aims to analyze observations' measures and perform a life cycle analysis (LCA) to evaluate the global environmental impacts linked to the implementation of the product DfSB strategies from a life cycle perspective.

3.6.1 Environmental Performance Measurements. For each observation, the global energy consumed for making one coffee was measured. This represented the primary variable for coffee maker environmental performance. The results obtained in the experiment show the evolution of energy consumption according to the user's profile (ES or NES) between observation $1(\mathrm{O} 1)$ and observation 2 or $3(\mathrm{O} 2$ or $\mathrm{O} 3)$ for each design intervention strategy (S1, S2, S3, and S4). Figure 7 shows that, in each case, the energy consumed for making one coffee was lower on the instrumented coffee makers than on the noninstrumented coffee makers.

A Wilcoxon signed rank test was used to analyze the significance of the environmental performance measurements. This test determines whether there is a statistically significant difference in the median of a dependent variable (here, the energy consumption (Wh)) when comparing two related groups (here, between $\mathrm{O} 1$ and $\mathrm{O} 2$ for each group). The test performed with MINITAB determined that there was a statistically significant median decrease in energy consumption for each group using instrumented coffee makers compared with the groups using noninstrumented coffee makers $(N=6 ; W=0 ; p<0.05)$, except for the ES group that tested the S4 prototype $(N=6, W=5, p=0.151)$ and the NES group that tested the $\mathrm{S} 1$ prototype $(N=6, W=3, p=0.142)$ (cf., Table 2)

To summarize, Fig. 7 suggests that including written information on the coffee makers (S4) results in significantly less energy being consumed by NES users (estimated median decrease in energy consumption $=-5.6 \mathrm{Wh}$ ), whereas for the ES users the reduction is not significant. Conversely, for the $\mathrm{S} 1$ prototype (ecofeedback), the reduction in energy consumption is significant for ES users (estimated median decrease in energy consumption $=-6.6 \mathrm{Wh}$ ) but not for NES users. Finally, for the last two prototypes (S2 and S3), a significant reduction in energy consumption can be observed. Table 2 provides a recap of these results.

In addition to these results, the same analysis was performed without differentiating user typologies (ES and NES) (Table 3). The pattern of results obtained shows that for each of the prototypes tested by the four groups of 12 participants, a significant decrease in energy consumption between $\mathrm{O} 1$ and $\mathrm{O} 2$ was observed. As shown in Fig. 7, for both profiles (ES and NES) the decrease in energy consumption between $\mathrm{O} 1$ and $\mathrm{O} 3$ is almost $50 \%$ compared to that of the reference coffee for S2. For the S1 strategy (eco-feedback), the results are also interesting $(-37 \%$ for ES and $-23 \%$ for NES). For the written information strategy (S4), the results in terms of reduced energy consumption during use are as follows: $-29 \%$ for ES and $-39 \%$ for NES compared to the reference coffee consumption. An analysis of the videos recorded during the observation phase showed that each strategy led to the espresso coffee maker switch-off time being reduced significantly (from $30 \%$ to $72 \%$ ) compared to the reference coffee. The reason for this environmental impact improvement is a change in user behavior: after making their coffee, the users remember to switch the coffee maker off.

3.6.2 Level of User Acceptance of the Strategies. As mentioned in Sec. 3.5, at the end of the experimentation, the users filled in a questionnaire in order to evaluate their perception of the design intervention strategy tested. The aim of this questionnaire is to assess their level of acceptance of the strategy in relation to their profile. The questionnaire provides a qualitative measurement of users' perception of the strategies regarding the following points:

- Strategy usefulness $(U \%)$

- Strategy efficiency $(E \%)$

- Strategy intrusiveness (I\%) 


\begin{tabular}{|c|c|c|c|c|}
\hline Strategy & $\begin{array}{l}\text { Eco-Feedback } \\
\text { (S1) } \\
\text { (Inform the user) }\end{array}$ & $\begin{array}{l}\text { Objective to reach (S2) } \\
\text { (Spur the user) }\end{array}$ & $\begin{array}{c}\text { Forcing Technology } \\
\text { (S3) } \\
\text { (Force the user) }\end{array}$ & $\begin{array}{c}\text { Written Information } \\
\text { (S4) } \\
\text { (Steer the user) }\end{array}$ \\
\hline Goal & $\begin{array}{l}\text { Provide the user a } \\
\text { feedback in real-time } \\
\text { regarding his energy } \\
\text { consumption }\end{array}$ & $\begin{array}{l}\text { Place user's energy } \\
\text { consumption on a gauge } \\
\text { to influence his behavior }\end{array}$ & $\begin{array}{l}\text { Automatic switch off } \\
\text { of the machine } \\
\text { immediately after } \\
\text { coffee realization }\end{array}$ & $\begin{array}{l}\text { Provide the user } \\
\text { instructions for saving } \\
\text { energy }\end{array}$ \\
\hline Prototype & $\begin{array}{c}\text { To realize your coffee, } \\
\text { you have consumed: } \\
14,5 \text { Wh } \\
\end{array}$ & \begin{tabular}{|c|} 
To realize vour coffee, you have \\
consumed: \\
$14,5 \mathrm{Wh}$ \\
The ideal energy consumption \\
for one coffee is $10 \mathrm{Wh}$
\end{tabular} & & $\begin{array}{c}\text { Please, switch off the } \\
\text { machine immediately } \\
\text { after use to save } \\
\text { energy }\end{array}$ \\
\hline $\begin{array}{c}\text { Technology } \\
\text { needed }\end{array}$ & $\begin{array}{l}\text { - Energysensor } \\
\text { - Screen }\end{array}$ & $\begin{array}{l}\text { - Energy sensor } \\
\text { Screen }\end{array}$ & $\begin{array}{l}\text { - Electronic } \\
\text { components }\end{array}$ & - Sticker \\
\hline
\end{tabular}

Fig. 5 Instrumentation of DfSB intervention strategies on Delonghi EC 150

Figure 8 suggests that strategies 1 and 2 (eco-feedback and objective, respectively) have the best score in terms of efficiency and usefulness compared to strategies 3 and 4 (forcing technology and written information). Moreover, the eco-feedback (S1) and objective strategies (S2) have the lowest score in terms of intrusiveness $(11.7 \%$ and $12 \%$, respectively) for ES users (against $25.7 \%$ and $41.7 \%$ for $\mathrm{S} 4$ and $\mathrm{S} 3$, respectively). The same tendency is observed for NES users: S1 and S2 strategies are perceived to be less intrusive than S3 and S4.

A Mann-Whitney test performed with MINITAB determined that there were, in majority, no statistically significant differences between the results of this questionnaire (usefulness, efficiency, and intrusiveness perception) for ES and NES users. The only exception here was the usefulness perception results for the ecofeedback strategy where the difference in score is significantly lower for NES users than for ES users $(N=6 ; W=44.5$; $p<0.05)$. The Mann-Whitney test determines whether there is a statistically significant difference between two unrelated, independent groups (here, the ES and NES groups) for a dependent variable (here, the median questionnaire score for the two groups).

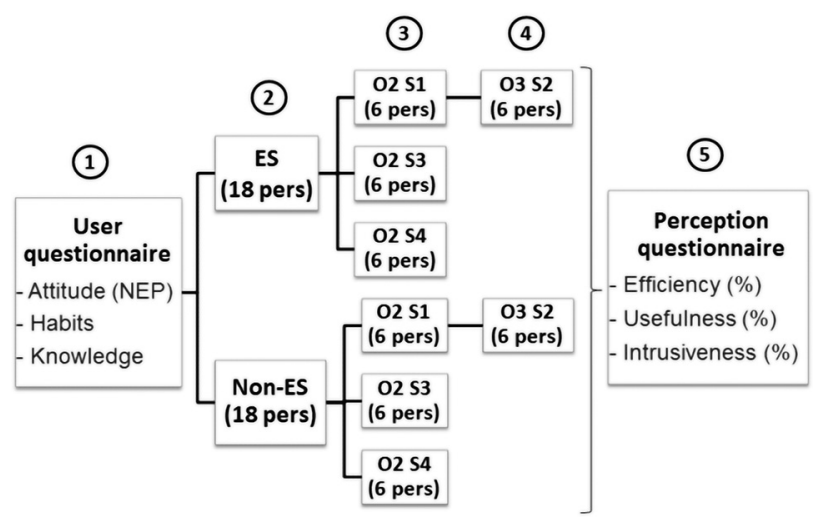

Fig. 6 Schematic diagram of the experimental observations (ES: eco-sensitive group, NES: non-eco-sensitive group, 01: observation 1, O2: observation 2, O3: observation 3, S1: ecofeedback strategy, S2: objective to reach strategy, S3: forcing technology strategy, and S4: written information strategy)
The high efficiency results for the eco-feedback strategy and the objective strategy (Fig. 7) together with the users' perception (Fig. 8) need to be compared with the environmental costs of these strategies from a global life cycle perspective. Section 3.6.3 presents the results of the LCA performed to analyze this aspect.

3.6.3 LCA Results. The LCA addresses the environmental aspects and potential environmental impacts of a product throughout its life cycle from the acquisition of raw materials to the production, use, end-of-life treatment, recycling, and final disposal. In this study, an LCA was performed with SIMAPRO 7.2 using the Eco indicator 99 method for impact calculation. The aim was to evaluate the added impacts related to the application of each strategy to the coffee maker and to compare these to the environmental benefits observed during use. The functional unit was specified as the preparation of two cups of coffee $(80 \mathrm{ml}$ each) per day, during $5 \mathrm{yr}$ (lifetime of the coffee maker). All life cycles phases, from raw material extraction to end of life, were considered in this analysis.

Based on the results presented in Fig. 7 (O1), the environmental impacts of the noninstrumented coffee maker were calculated according to Eq. (1) (LCA 1). The results of this first LCA are used as a reference for comparison with the instrumented makers (cf., Table 4).

For each strategy, $\Delta \mathrm{IE}_{\text {global }}(2)$ was calculated based on the studies performed on the NES and ES groups. For this analysis, the mean value of energy consumption reduction presented in Fig. 7 was taken as a use scenario for each profile and each strategy. The gains in use were compared with the environmental losses associated with other life cycle phases due to the technology added to implement the strategies. Table 4 presents these results.

It can be seen that the environmental impacts decrease because of the new usage of the product and to the technical solution implemented. This technical solution has practically no impact on the entire product life cycle for strategy S4 (written information). For other strategies like S1, the impacts transferred to other life cycle phases are too significant to be compensated by the environmental gains during use. The results for strategy S3 are interesting because only three indicators are deteriorated. These results can be linked with those presented in Sec. 3.6.2 (level of acceptability). Designers must compare the LCA results obtained using a global life cycle vision of the strategies implemented with the level of acceptability of the strategy as perceived by the users in order to choose most viable solutions. They need to decide on a 

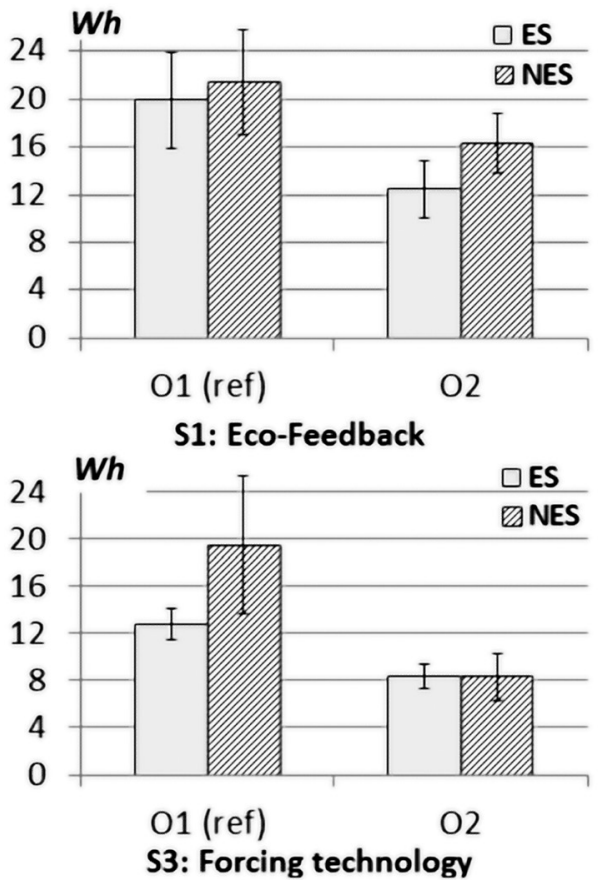

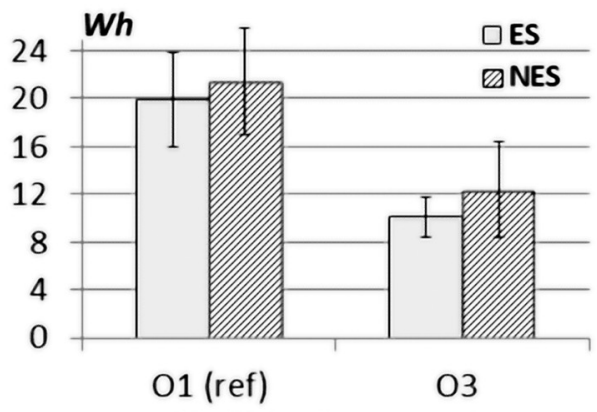

S2: Objective to reach

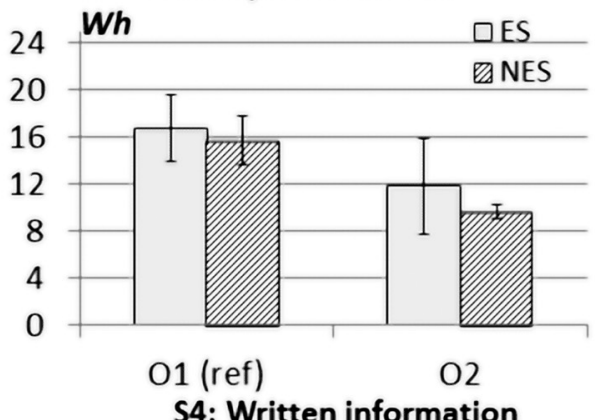

Fig. 7 Energy consumption (Wh) for making one coffee on a regular coffee machine (01) compared to an instrumented coffee machine $(\mathrm{O} / \mathrm{O} / \mathrm{3})$ for two different profiles of users (ES: ecosensitive group and NES: non-eco-sensitive group)

Table 2 Significance of energy decrease between 01 and 02 depending on user profile

\begin{tabular}{|c|c|c|c|c|}
\hline Prototype tested & Group ested & $\begin{array}{l}\text { Wilcoxon signed rank } \\
\text { test results }\end{array}$ & $\begin{array}{l}\text { Estimated median decrease in energy } \\
\text { consumption between } \mathrm{O} 1 \text { and } \mathrm{O} 2 \text { (Wh) }\end{array}$ & Significance \\
\hline \multirow[t]{2}{*}{ Eco-feedback (S1) } & ES & $(N=6 ; W=0 ; p=0.036)$ & -6.6 & Yes \\
\hline & NES & $(N=6 ; W=3 ; p=0.142)$ & -3.7 & No \\
\hline \multirow[t]{2}{*}{ Objective to reach (S2) } & ES & $(N=6 ; W=0 ; p=0.036)$ & -10.3 & Yes \\
\hline & NES & $(N=6 ; W=0 ; p=0.036)$ & -7.3 & Yes \\
\hline \multirow[t]{2}{*}{ Forcing technology (S3) } & ES & $(N=6 ; W=0 ; p=0.036)$ & -4.3 & Yes \\
\hline & NES & $(N=6 ; W=0 ; p=0.036)$ & -9.8 & Yes \\
\hline \multirow[t]{2}{*}{ Written information (S4) } & ES & $(N=6 ; W=5 ; p=0.151)$ & -4.7 & No \\
\hline & NES & $(N=6 ; W=0 ; p=0.036)$ & -5.6 & Yes \\
\hline
\end{tabular}

Table 3 Significance of energy decrease between 01 and 02 regardless of user profile

\begin{tabular}{lrrr}
\hline \hline Prototype tested & $\begin{array}{c}\text { Wilcoxon signed } \\
\text { rank test results }\end{array}$ & $\begin{array}{c}\text { Estimated median decrease in energy } \\
\text { consumption between O1 and O2 (Wh) }\end{array}$ & Significance \\
\hline Eco-feedback (S1) & $(N=12 ; W=2 ; p=0.007)$ & -5.1 & -10.0 \\
Objective to reach (S2) & $(N=12 ; W=0 ; p=0.003)$ & -6.7 & Yes \\
Forcing technology (S3) & $(N=12 ; W=0 ; p=0.003)$ & Yes \\
Written information (S4) & $(N=12 ; W=10 ; p=0.014)$ & Yes \\
\hline \hline
\end{tabular}

compromise since, in some cases, a strategy that performs well from a life cycle point of view may not be accepted by the user and therefore will not be efficient over time owing to this low level of acceptability.

\section{Discussion}

The proposed protocol examines ways of improving the environmental performance of a consumer product use phase by modifying user behavior through the implementation of design intervention strategies. This protocol is built on a microlevel analysis of the use phase and is supported by product-in-use observations to capture user behavior in a real-life context. The DfSB strategy approach with its estimation of environmental impacts on the global life cycle of the product provides designers with new information allowing them to efficiently implement use-related behavior innovation in a product's design. The realization of an LCA at the end of the protocol brings new elements to designers for the identification of environmentally relevant solutions.

The main finding from the case study presented is that the design of a product can indeed induce changes in user behavior, encouraging that behavior to become more sustainable. Focusing on product design, therefore, seems to be a feasible way to induce and maintain sustainable user behavior and use-related environmental performance. Indeed, all the design strategies implemented on the coffee maker had a significant positive effect on the reduction of environmental impacts during use via the modification of the users' behavior (regardless of their eco-profile). The statistical 


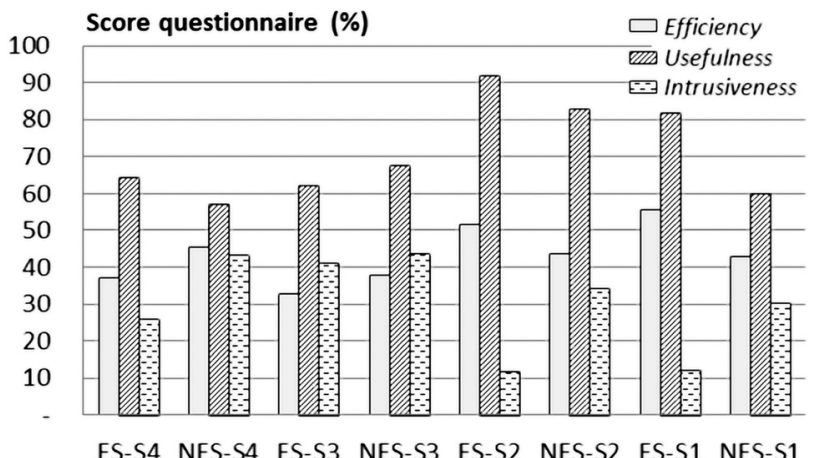

Fig. 8 Perception of the different strategies (efficiency, usefulness, and intrusiveness) according to user's profile. (ES: eco-sensitive group, NES: non-eco-sensitive group, S1: ecofeedback strategy, S2: objective to be reached strategy, S3: forcing technology strategy, and S4: written information strategy).

analysis performed only showed a significant behavioral difference between the two types of user profiles (ES versus NES) when the forcing strategy (S2) was implemented. For other strategies, it was not possible to demonstrate that the decrease in energy consumption was more significant for one profile or the other. First, this may be due to the small size of the panel of participants in our experiment. Indeed, there was no difference in the behavior of both profile types in step 3 of our protocol. Observation of a larger panel of participants may provide more generalized results. Second, the lack of difference may also be due to the fact that the variables measured in order to determine the user profiles were not relevant in terms of splitting them into groups and explaining and understanding their behavior. Other variables measured in the prequestionnaire (such as age, gender and socioprofessional category) should be analyzed to determine potential correlations with use-related behavior in order to refine the environmental profile categorization.

Another interesting finding from this experiment is that the environmental impacts of the global life cycle of the product can be improved when the user's interaction with the product is taken into account in the design activities. In order to do this, the results presented in Table 4 (LCA results) are linked with the user strategy acceptability results presented in Fig. 3. This link helps to define the efficient design intervention strategies for inducing sustainable behavior based on the best compromise between environmental gains during use, impact transfers to other life cycle phases, and the level of acceptability of the strategy for the users. The final decision will depend on the company's strategy, the resources available, and other design criteria.

In the proposed research protocol, the question of DfSB strategy efficiency over time is not integrated. To further this research, the potential loss of DfSB strategy efficiency over time due to wear on the solution needs to be taken into account. Some researchers have demonstrated that a message or a strategy that aims to induce sustainable behavior becomes less efficient over time. Models forecasting the performance of these strategies have been created [16]. Product upgradability can in fact help resolve this aspect by gradually integrating into product design solutions encouraging the user to use a product efficiently.

\section{Conclusion and Future Work}

The research protocol proposed in this paper aims to support eco-design activities for the product use phase through use behavior innovations. Based on studying the use phase of the product and interactions between user variables that influence behavior, usage context, and environmental impacts during use, this protocol helps identify efficient design strategies able to substantially improve environmental performance during product use. The use of an eco-design approach and a multicriteria and multiphase vision for the selection of relevant DfSB strategies has been integrated in this approach in order to support the decisions taken during the design process for efficient implementation of DfSB strategies.

The case study presented demonstrates that user variables regulating behavior during use can to be taken into consideration in eco-design approaches in order to understand how to reduce environmental impacts in use. The inclusion of a global life cycle vision in this procedure represents a new dimension in DfSB approaches. If designers only look at the efficiency of DfSB strategies during product use, they may well make the wrong decision in terms of design solutions. The protocol presented compares the potential use-related environmental gains offered by design solutions according to user profile with the environmental losses measured on other life cycle phases. Taking into account the level of acceptability of strategies for users provides designers with better information: it allows them to choose the best compromise between the aforementioned three aspects and to identify which design solutions are the best.

Owing to the difficulty in implementing this protocol in a traditional design process, the next steps involve developing a tool based on the methodological elements presented in this paper. The aim is then to support the application of such a tool in industry. The tool will allow designers to identify product usage drifts based on an automatic questionnaire. The results of this stage will then allow them to choose the right DfSB strategies from an environmental point of view. The tool might take on the format of an Excel file that analyzes designers' answers to step 1 of the protocol. Designers will then be presented with a list of design recommendations based on the usage drifts identified using the tool. This will help them to choose the relevant DfSB strategies to be implemented on the product. Future work will also involve

Table 4 LCA results

\begin{tabular}{|c|c|c|c|c|c|c|c|c|c|}
\hline \multirow[b]{2}{*}{ Environmental indicators } & \multirow{2}{*}{$\begin{array}{c}\text { Ref. } \\
\text { LCA1 }(\%)\end{array}$} & \multicolumn{2}{|c|}{$\Delta \mathrm{IE} \mathrm{S} 1$ (ecofeedback) } & \multicolumn{2}{|c|}{$\Delta \mathrm{IE} \mathrm{S} 2$ (objective) } & \multicolumn{2}{|c|}{$\Delta \mathrm{IE} \mathrm{S3}$ (forcing tech.) } & \multicolumn{2}{|c|}{$\Delta \mathrm{IE} \mathrm{S} 4$ (written info) } \\
\hline & & $\mathrm{ES}(\%)$ & $\operatorname{NES}(\%)$ & $\mathrm{ES}(\%)$ & $\operatorname{NES}(\%)$ & $\mathrm{ES}(\%)$ & $\operatorname{NES}(\%)$ & $\mathrm{ES}(\%)$ & NES $(\%)$ \\
\hline Carcinogens & 100 & +46.5 & +55.3 & +42.1 & +46.5 & +37.7 & +28.3 & -18.2 & -22.6 \\
\hline Respiratory organics & 100 & +2.0 & +4.4 & +0.9 & +2.0 & 0.0 & -2.2 & -4.6 & -5.9 \\
\hline Respiratory inorganics & 100 & +4.0 & +8.5 & +1.5 & +4.0 & -0.5 & -5.0 & -9.5 & -11.6 \\
\hline Climate change & 100 & -2.9 & +4.2 & -6.4 & -2.9 & -6.7 & -13.8 & -14.2 & -17.6 \\
\hline Radiation & 100 & -38.7 & -19.0 & -48.4 & -38.7 & -38.7 & -58.5 & -39.5 & -49.2 \\
\hline Ozone layer & 100 & -34.0 & -15.4 & -43.1 & -34.0 & -35.1 & -53.7 & -36.2 & -45.7 \\
\hline Ecotoxicity & 100 & +20.0 & +21.0 & +18.9 & +20.0 & +13.8 & +12.7 & -3.6 & -4.6 \\
\hline Acidification/eutrophication & 100 & +1.0 & +5.8 & -1.5 & +1.0 & -2.6 & -7.6 & -9.9 & -12.2 \\
\hline Land use & 100 & -33.1 & -14.7 & -42.2 & -33.1 & -33.4 & -51.9 & -36.6 & -45.6 \\
\hline Minerals & 100 & +28.6 & +28.6 & +28.6 & +28.6 & +6.3 & +6.3 & 0.0 & 0.0 \\
\hline Fossil fuels & 100 & -0.4 & +4.4 & -2.6 & -0.4 & -2.6 & -7.0 & -9.2 & -11.4 \\
\hline
\end{tabular}

Values in bold represent environmental losses 
building a database of DfSB strategies. It will include usage drifts identified and typology of product considered together with the associated environmental costs. Finally, the question of strategy efficiency over time could also be integrated into the tool based on the various examples found in the literature and existing algorithms.

\section{Acknowledgment}

This work was part of the IDCyclUM project (Design and Integration Methodology for Sustainable Innovation with Multiple Upgrade Cycles) funded by the French Research Agency (Ref: ANR-11-EC-OT-0001).

\section{References}

[1] Yannou, B., Jankovic, M., Leroy, Y., and Okudan Kremer, G. E., 2013, "Observations From Radical Innovation Projects Considering the Company Context," ASME J. Mech. Des., 135(2), p. 021005.

[2] Tukker, A., and Jansen, B., 2006, "Environmental Impacts of Products: A Detailed Review of Studies," J. Ind. Ecol., 10(3), pp. 159-182.

[3] Brundtland, G. H., 1987, Our Common Future, Oxford University Press, Oxford, UK.

[4] Cor, E., Domingo, L., Brissaud, D., and Zwolinski, P., 2014, "A Protocol to Perform Usage Oriented Ecodesign,” CIRP Ann. Manuf. Technol., 63(1), pp. 169-172.

[5] Helu, M., Vijayaraghavan, A., and Dornfeld, D.,2011, "Evaluating the Relationship Between Use Phase Environmental Impacts and Manufacturing Process Precision," CIRP Ann. Manuf. Technol., 60(1), pp. 49-52.

[6] Guinée, J., Heijungs, R., De Koning, A., Van, L., Geerken, T., Van Holderbeke, M., and Delgado, L., 2006, "Environmental Impact of Products (EIPRO) Analysis of the Life Cycle Environmental Impacts Related to the Final Consumption of the EU25," Report No. EUR22284EN.

[7] Plouffe, S., Lanoie, P., Berneman, C., and Vernier, M.-F., 2011, "Economic Benefits Tied to Ecodesign," J. Cleaner Prod., 19(6-7), pp. 573-579.

[8] Sauer, J., Wiese, B. S., and Rüttinger, B., 2003, "Designing Low-Complexity Electrical Consumer Products for Ecological Use," Appl. Ergon., 34(6), pp. 521-531.

[9] ISO, X., 2003, XP ISO/TR 14062: Environmental Management_Integrating Environmental Aspects into Product Design and Development, Geneva, Switzerland.

[10] Ramani, K., Ramanujan, D., Bernstein, W. Z., Zhao, F., Sutherland, J., Handwerker, C., and Thurston, D., 2010, "Integrated Sustainable Life Cycle Design: A Review," ASME J. Mech. Des., 132(9), p. 091004.

[11] Bohm, M. R., Haapala, K. R., Poppa, K., Stone, R. B., and Tumer, I. Y., 2010, "Integrating Life Cycle Assessment Into the Conceptual Phase of Design Using a Design Repository," ASME J. Mech. Des., 132(9), p. 091005.

[12] Serna-Mansoux, L., Domingo, L., Millet, D., and Brissaud, D., 2014, "A Tool for Detailed Analysis and Ecological Assessment of the Use Phase," Procedia CIRP, 15, pp. 502-507.

[13] Sauer, J., Wiese, B. S., Ruttinger, B., 2002, "The Utilisation Phase as a Critical Element in Ecological Design," Mechanical Life Cycle Handbook: Good Environmental Design and Manufacturing, M. S. Hundal, ed., New York.

[14] Seow, Y., and Rahimifard, S., 2011, "A Framework for Modelling Energy Consumption Within Manufacturing Systems," CIRP J. Manuf. Sci. Technol., 4(3), pp. 258-264.

[15] Behdad, S., Kwak, M., Kim, H., and Thurston, D., 2010, "Simultaneous Selective Disassembly and End-Of-Life Decision Making for Multiple Products that Share Disassembly Operations," ASME J. Mech. Des., 132(4), p. 041002.

[16] Serna-Mansoux, L., Popoff, A., and Millet, D., 2014, "A Simplified Model to Include Dynamic Product User Interaction in the Eco-Design Process," J. Ind. Ecol., 18(4), pp. 529-544.

[17] Huang, H., and Ameta, G., 2014, "A Novel Pattern for Energy Estimation Framework and Tools to Compute Energy Consumption in Product Life Cycle," ASME J. Comput. Inf. Sci. Eng., 14(1), p. 011002.
[18] European Commission, 2010, "DIRECTIVE 2009/125/EC: Establishing a Framework for the Setting of Ecodesign Requirements for Energy-Related Products, Luxemborg.

[19] Kuijer, L., and De Jong, A., 2012, "Identifying Design Opportunities for Reduced Household Resource Consumption: Exploring Practices of Thermal Comfort,” J. Des. Res., 10(1), pp. 67-85.

[20] Srivastava, J., and Shu, L. H., 2013, "Affordances and Product Design to Support Environmentally Conscious Behavior,” ASME J. Mech. Des., 135(10), p. 101006

[21] Lidman, K., Renström, S., and Karlsson, M., 2011, "The Green User. Design for Sustainable Behaviour," Proceedings From the IASDR Conference 2011 Diversity and Unity, Oct. 31-Nov. 1, Delft, pp. 1-12.

[22] Ajzen, I., 1991, "The Theory of Planned Behavior," Organ. Behav. Hum. Decis. Processes, 50(2), pp. 179-211.

[23] Abrahamse, W., Steg, L., Vlek, C., and Rothengatter, T., 2005, "A Review of Intervention Studies Aimed at Household Energy Conservation," J. Environ. Psychol., 25(3), pp. 273-291.

[24] Srivastava, J., and Shu, L. H., 2013, "Encouraging Resource-Conscious Behavior Through Product Design: The Principle of Discretization," ASME J. Mech Des., 135(6), p. 061002.

[25] Lilley, D., 2009, "Design for Sustainable Behaviour: Strategies and Perceptions," Des. Stud., 30(6), pp. 704-720.

[26] Gustafsson, A., and Gyllenswärd, M., 2005, "The Power-Aware Cord: Energy Awareness Through Ambient Information Display," Proceedings of the CHI'05 Extended Abstracts on Human Factors in Computing Systems, pp. 1423-1426.

[27] Midden, C. J. H., Meter, J. E., Weenig, M. H., and Zieverink, H. J. A., 1982 "Using Feedback, Reinforcement and Information to Reduce Energy Consumption in Households: A Field-Experiment," J. Econ. Psychol., 3(1), pp. 65-86.

[28] Wood, G., and Newborough, M., 2003, "Dynamic Energy-Consumption Indicators for Domestic Appliances: Environment, Behaviour and Design,” Energy Build., 35(8), pp. 821-841.

[29] Withanage, C., Ashok, R., Hölttä-Otto, K., and Otto, K., 2014, "Identifying and Categorizing Opportunities for Design for Sustainable User Behavior," ASME Paper No. V007T07A008.

[30] Bhamra, T., Lilley, D., and Tang, T., 2011, "Design for Sustainable Behaviour: Using Products to Change Consumer Behaviour," Des. J., 14(4), pp. 427-445.

[31] Wever, R., van Kuijk, J., and Boks, C., 2008, "User-Centred Design for Sustainable Behaviour," Int. J. Sustainable Eng., 1(1), pp. 9-20.

[32] Boks, C., 2012, "Design for Sustainable Behaviour Research Challenges," Design for Innovative Value Towards a Sustainable Society, Springer, Amsterdam, The Netherlands, pp. 328-333.

[33] Hines, J. M., Hungerford, H. R., and Tomera, A. N., 1987, "Analysis and Synthesis of Research on Responsible Environmental Behavior: A Meta-Analysis," J. Environ. Educ., 18(2), pp. 1-8.

[34] Fishbein, M., and Ajzen, I., 1975, Belief, Attitude, Intention, and Behavior: An Introduction to Theory and Research, Addison-Wesley, Boston, MA.

[35] Maguire, M., 2001, "Methods to Support Human-Centred Design," Int. J. Hum. Comput. Stud.," 55(4), pp. 587-634.

[36] Strömberg, H., Selvefors, A., and Renström, S., "Mapping Out the Design Opportunities: Pathways of Sustainable Behaviour," Int. J. Sustainable Eng. Available at: http://publications.lib.chalmers.se/publication/211918-mapping-outthe-design-opportunities-pathways-of-sustainable-behaviour

[37] Evans, S., Burns, A., and Barrett, R., 2002, Empathic Design Tutor, IERC, Cranfield University, Cranfield, UK.

[38] Quantis Suisse, 2011, "Comparative Full Life Cycle Assessment of B2C Cup of Espresso Made Using a Packaging and Distribution System From Nespresso Espresso and Three Generic Products," Final Report No. 3.

[39] Wimmer, W, and Züst, R., 2003, ECODESIGN Pilot: Product-Investigation Learning-and Optimization-Tool for Sustainable Product Development With CDROM, Vol. 3, Springer Science \& Business Media, Dordrecht, The Netherlands.

[40] European Commission (DG ENER), 2011, Preparatory Study for Eco-Design Requirements of EuPs-Lot 25: Non-Tertiary Coffee Machines, Paris, France.

[41] Dunlap, R. E., Van Liere, K. D., Mertig, A. G., and Jones, R. E., 2000, "New Trends in Measuring Environmental Attitudes: Measuring Endorsement of the New Ecological Paradigm: A Revised NEP Scale," J. Soc. Issues, 56(3), pp. 425-442. 